\title{
Radiotherapy for large cutaneous angiosarcoma of face with RapidArc (VMAT)
}

\author{
Mirza Athar Ali, Babaiah Muntimadugu, Madhusudhan Nagaraju, Sujana Priya Vuba, \\ Geomcy George, Kanaparthy Raja Muralidhar, Birendra Kumar Rout, Prabhakar Mariappan \\ Department of Radiation Oncology, American Oncology Institute, Hyderabad, India
}

Received December 10, 2014; Revised January 30, 2015; Accepted February 04, 2015; Published Online February 06, 2015

\section{Case Report}

\begin{abstract}
Angiosarcoma is a rare malignancy of vascular origin. It can affect any part of the body, head and neck region being probably the most common site of diagnosis. We present here a case of Angiosarcoma of face in a 67-year-old elderly gentleman who was treated with RapidArc - volumetric modulated arc therapy (VMAT) for recurrence after surgery, radiotherapy and chemotherapy. As an alternative to Electron Beam Therapy, RapidArc with skin bolus can be considered for large complex shaped targets with irregular surface and tissue inhomogeneity. RapidArc plan can achieve adequate target coverage with acceptable dose homogeneity and conformity.
\end{abstract}

Keywords: RapidArc; Cutaneous Angiosarcoma

\section{Introduction}

Angiosarcoma is a rare malignancy of vascular origin and accounts for approximately $2 \%$ of soft tissue sarcomas. It can affect any part of the body, one third being cutaneous and one fourth being of soft tissue origin. These tumours arise from vascular endothelium, show multifocality and can spread to lymph nodes and distant sites. The peak age of incidence appears to be seventh decade and men are affected more than women. Causes of angiosarcoma include therapeutic radiation, exposure to vinyl chloride and chronic lymphedema (Stewart-Treves syndrome). ${ }^{1}$ Head and neck region is probably the most common site of diagnosis and there is a predilection for sun exposed skin of scalp in older individuals. Head and neck angiosarcoma morphologic appearance is somewhat distinct from other locations by its common association with a heavy lymphocytic infiltrate that can obscure its findings. ${ }^{2}$ Angiosarcoma expresses the typical vascular markers: CD34, CD31, Fli-1 and ERG. ${ }^{3,4}$

Standard treatment for angiosarcoma includes surgical excision with clear margins followed by local radiotherapy with or without systemic chemotherapy.

\section{Case presentation}

A 67-year-old gentleman with no medical co-morbidities, presented to us in September 2012 with 3 months history of ulcerative growth over right ala of nose, which started as a small nodule which eventually grew and ulcerated with bloody discharge. On clinical examination, there was evidence of a nodular-ulcerated lesion measuring $4 \times 3 \mathrm{~cm}$ over the right ala of the nose with uneven surface, irregular diffuse margins and indurated erythematous skin over the right cheek adjacent to the lesion.

There was no evidence of palpable cervical lymphadenopathy. Skin biopsy showed evidence of poorly differentiated carcinoma. Patient underwent wide local excision with flap reconstruction.

The post-operative histopathology and Immunohistochemistry IHC study was suggestive of angiosarcoma with positive resected margins. Patient received post-operative radiation therapy to a dose of $60 \mathrm{~Gy}$ in 30 fractions with intensity modulated radio therapy IMRT in October-November, 2012. Subsequently, he was started on adjuvant chemotherapy with inj. Paclitaxel. Patient developed local recurrence characterized by erythematous-bullous lesion over the right zygomatic region (Figure 1) in the month of July 2013 immediately after completing 5 cycles of adjuvant chemotherapy. 


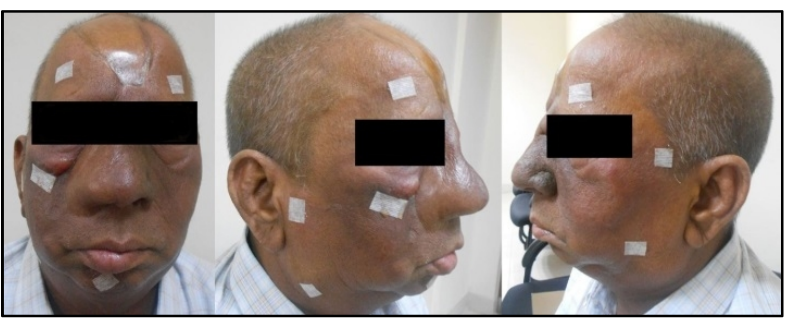

FIG. 1: Multiple cutaneous biopsies were taken to map the microscopic spread of the disease. Right zygomatic region shows recurrent lesion.

\section{Imaging findings}

Preoperative whole-body positron emission tomography (PET-CT) scan revealed evidence of $3 \times 2 \mathrm{~cm}$ metabolically active irregular soft tissue mass in right nasal ala with SUV Max of 10.3 with no evidence of any nodal or distant metastasis. Whole-body PET CT performed 3 months post radiation therapy showed no evidence of any locoregional recurrence or distant metastasis. PET CT performed 7 months post radiation therapy (Figure 2) revealed evidence of locoregional recurrence characterized by:

1. Intense FDG uptake with Standardized Uptake Value (SUV) Max of 12.7 in a plaque like area in the skin and subcutaneous tissue over the right maxillary and zygomatic region involving right lower eyelid and right medial canthus.

2. Another intense FDG uptake with SUV Max of 11 in a similar area of skin thickening on the left side of the face involving left medial canthus and upper zygomatic region.

3. Another focus of intense FDG uptake with SUV Max of 10 in the area of skin thickening in the right buccal region.

4. Focal increased FDG uptake with SUV Max of 7 in the nasal bridge just above the level tip of nose.

5. No evidence of metabolically active disease elsewhere to suggest distant metastasis.

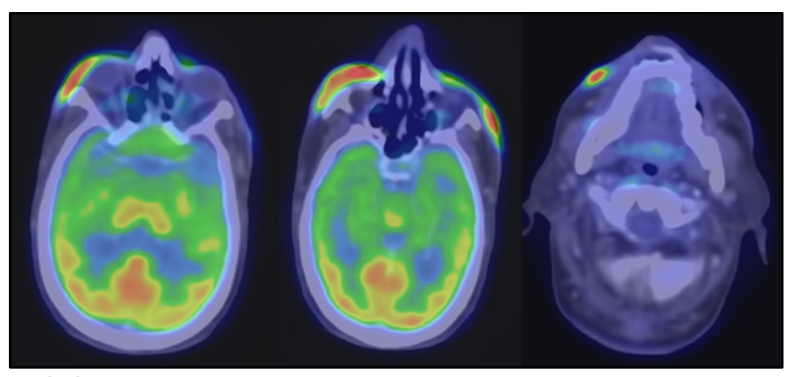

FIG. 2: PET CT scan axial images showing metabolically active recurrent lesions.

Biopsy from the skin confirmed it as recurrent angiosarcoma. Second surgery was deferred by the head and neck surgeon in view of his elderly age and difficulty in achieving negative margins considering the local extent and multifocality of the disease on PET-CT. Since the disease was chemotherapy refractory, he was referred to us for re-irradiation as the only available option. To map the local extent of the disease, he underwent multiple cutaneous biopsies $4-5 \mathrm{~cm}$ from the periphery of documented recurrent lesions (Figure 1). All the seven biopsy specimens were negative for malignancy.

We simulated this patient on a 16-slice PET-CT simulator. Patient was immobilized using a thermoplastic mask in neutral head position. CT images were acquired in $1.25 \mathrm{~mm}$ slice thickness from vertex to mid chest. PET-CT fusion was performed using Eclipse version 10.0 software. Gross tumor volume (GTV) included FDG avid PET positive disease and clinical target volume (CTV) included GTV $+3 \mathrm{~cm}$ margin to incorporate microscopic disease. CTV was cropped from the adjacent organs at risk (OARs). Planning target volume (PTV) included CTV $+0.5 \mathrm{~cm}$ margin for setup errors. Prescription dose was $60 \mathrm{~Gy}$ in 30 fractions to the PTV.

\section{Discussion}

For superficial tumours of skin and subcutaneous tissue, electron beam therapy is an ideal modality of radiation therapy. One of the basic requirements for electron beam therapy is presence of relatively flat or symmetric treatment area in order to reduce chances of excessive dose inhomogeneity. Sharp surface irregularities where the electron beam may be incident tangentially give rise to a complex dose distribution with localized over-dose (hot-spot) and under-dose (cold-spot). ${ }^{5}$ In our case, the PTV included almost entire face which made it extremely difficult to be treated with electron beam, considering the surface irregularity and tissue inhomogeneity. Hence, we decided to treat this patient with RapidArc (Volumetric Modulated Arc Therapy-VMAT) 6, 7,8 using 0.5 centimetres wax bolus over the thermoplastic cast (Figure 3) to bring the prescribed isodose up to the level of skin surface. Zacarias et al. ${ }^{9}$ used VMAT for superficial targets and could achieve improved dose uniformity close to skin surface. Treatment planning was done using two co-planar arcs with 360 degree rotation. $0.5 \mathrm{~cm}$ tissue equivalent bolus over the PTV was used for planning purpose (Figure 4A-E). In-vivo dose measurement was done to confirm the superficial dose build-up. Figure 5 shows brisk cutaneous reaction during the course of treatment. Dose homogeneity index $\left[\mathrm{I}_{\max } / \mathrm{I}_{\mathrm{ref}}\right]{ }^{10,11}$ was 1.09 and conformity index $\left[\mathrm{V}_{\text {ref }} / \mathrm{V}_{\mathrm{T}}\right]^{11}$ was $1.04 .97 \%$ of the PTV received $100 \%$ of the prescribed dose.

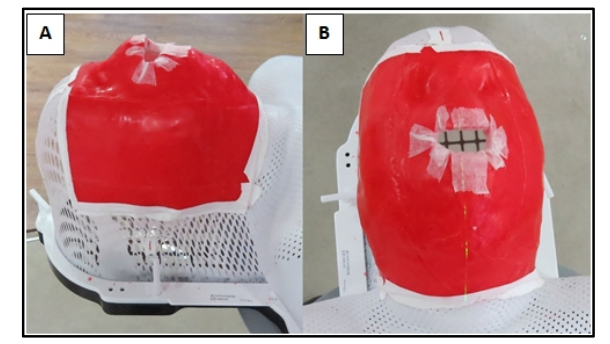

FIG. 3: Thermoplastic mask with $0.5 \mathrm{~cm}$ custom wax bolus for surface dose build-up. 

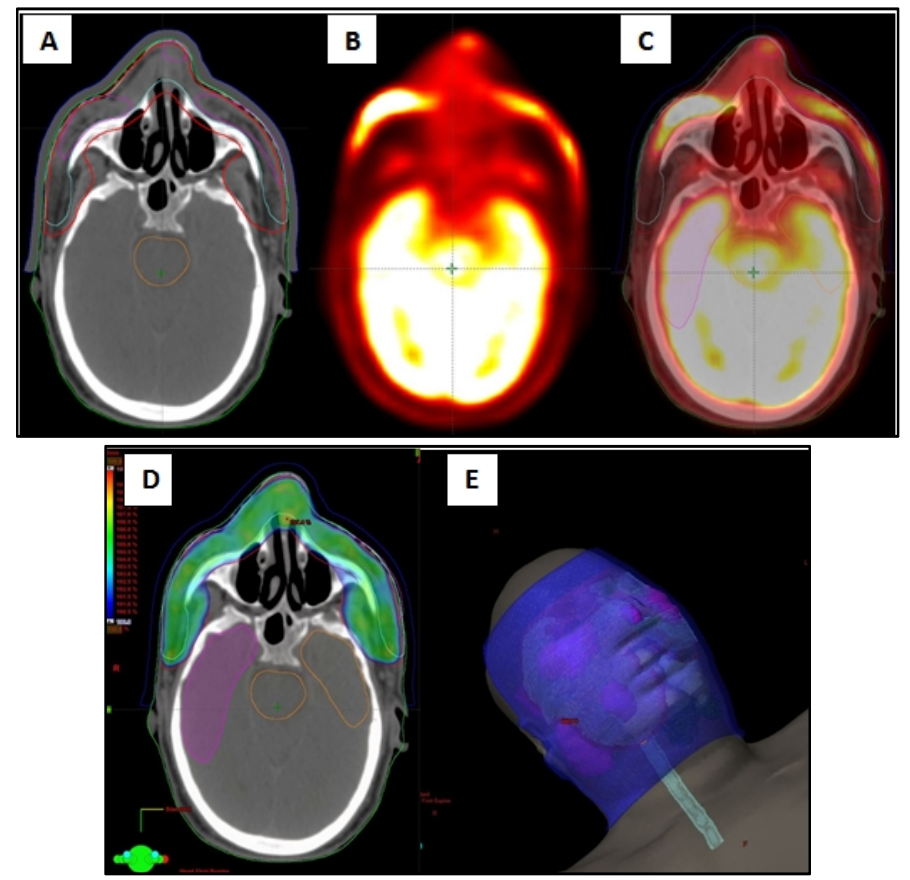

FIG. 4: A: Simulation CT showing PTV contour (red), CTV (cyan), GTV (magenta) and custom bolus over the skin (grey); B: PET image showing FDG avid lesion over the right, left zygomatic region and nasal bridge; C: PET-Planning CT fusion, D: Dose distribution in color wash showing PTV coverage and dose build-up on the skin surface with custom bolus; E: 3-dimentional rooms-eye-view of patient and custom bolus.

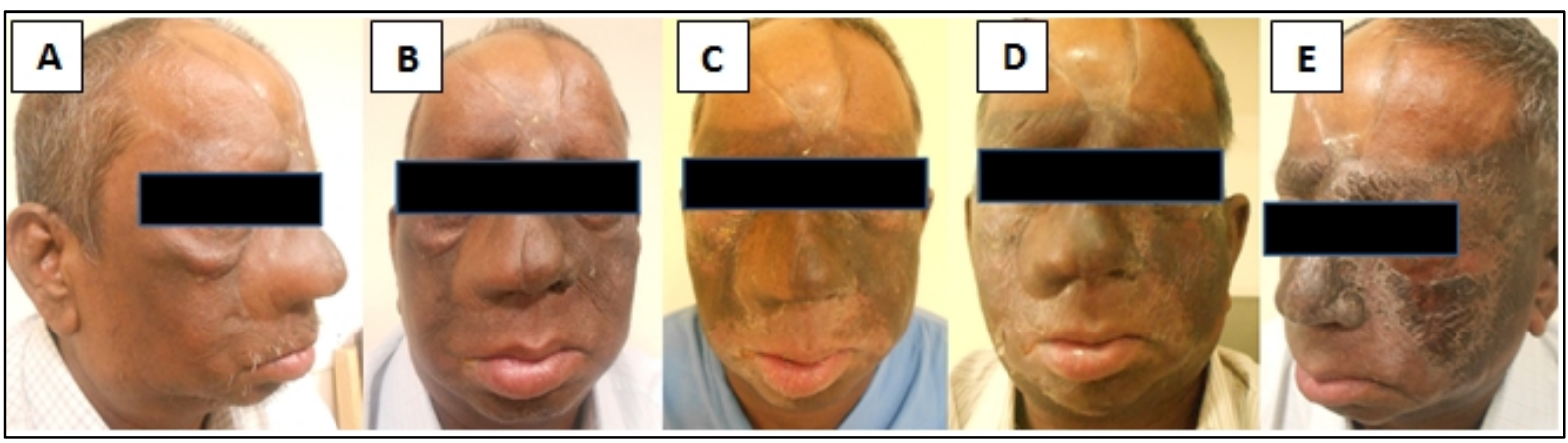

FIG. 5: Clinical photographs during the course of treatment showing brisk cutaneous reaction due to adequate cutaneous dose build-up. A: $2^{\text {nd }}$ week; B: $3^{\text {rd }}$ week; C: $4^{\text {th }}$ week; D: $5^{\text {th }}$ week; and E: $6^{\text {th }}$ week of radiotherapy.

\section{Conclusion}

Radiotherapy for large cutaneous angiosarcoma of face is challenging considering the surface irregularity and tissue inhomogeneity. As an alternative to electron beam therapy, RapidArc (VMAT) can be considered for such cases using tissue equivalent bolus for cutaneous build-up of the dose. RapidArc (VMAT) can achieve reasonable dose homogeneity and conformity with adequate target coverage.

\section{Conflict of interest}

The authors declare that they have no conflicts of interest. The authors alone are responsible for the content and writing of the paper.

\section{References}

1. Stewart FW, Treves N. Lymphangiosarcoma in post-mastectomy lymphedema: a report of six cases in elephantiasis chirurgica. Cancer 1948; 1:64-81.

2. Antonescu C. Malignant vascular tumors - An update. Modern Pathology 2014; 27: S30-8.

3. Miettinen M, Lindenmayer AE, Chaubal A. Endothelial cell markers CD31, CD34 and BHN-9, antibody to $\mathrm{H}$ - and $\mathrm{Y}$-antigens - evaluation of their specificity and sensitivity in the diagnosis of vascular tumors and comparison with von Willebrand factor. Mod Pathol 1994; 7:82-90.

4. Folpe AL, Chand EM, Goldblum JR, Weiss SW. Expression of Fli-1, a nuclear transcription factor, 
distinguishes vascular neoplasms from potential mimics. Am J Surg Pathol 2001; 25:1061-6.

5. Khan FM, Doppke KP, Hogstrom KR, et al. Clinical electron-beam dosimetry: report of AAPM Radiation Therapy Committee Task Group No. 25. Med Phys 1991; 18:73-109.

6. Ali MA, Babaiah M, Madhusudhan N, George G, Jain S, Ramalingam K, Kumar SA, Karthikeyan K, Anantharaman A . Comparative dosimetric analysis of IMRT and VMAT (RapidArc) in brain, head and neck, breast and prostate malignancies. Int J Cancer Ther Oncol 2015; 3: 03019.

7. Rout BK, Muralidhar KR, Ali M, Shekar MC, Kumar A. Dosimetric study of RapidArc plans with flattened beam (FB) and flattening filter-free (FFF) beam for localized prostate cancer based on physical indices. Int J Cancer Ther Oncol 2014; 2:02046.

8. Rana S, Pokharel S, Zheng Y, Zhao L, Risalvato D, Vargas C, Cersonsky N. Treatment planning study comparing proton therapy, RapidArc and intensity modulated radiation therapy for a synchronous bilateral lung cancer case. Int I Cancer Ther Oncol 2014; 2:020216.
9. Zacarias AS, Brown MF, Mills MD. Volumetric modulated Arc therapy (VMAT) treatment planning for superficial tumors. Med Dosim 2010; 35:226-9.

10. Collins SP, Coppa ND, Zhang Y, et al. Cyberknife radiosurgery in the treatment of complex skull base tumours: analysis of treatment planning parameters. Radiat Oncol 2006; 1:46.

11. Feuvret L, Noël G, Mazeron JJ, Bey P. Conformity index: a review. Int J Radiat Oncol Biol Phys 2006; 64:333-42. 